\section{MS6-P10 Crystal structure of the human IL-17AF heterodimer}

Arnaud Goepfert ${ }^{1}$, Sylvie Lehmann ${ }^{1}$, Jean-Michel Rondeau ${ }^{1}$

1. Novartis Institutes for BioMedical Research, Basel, Switzerland

email: arnaud.goepfert@novartis.com

IL-17A and IL-17F are the best characterized and most closely-related members of the IL-17 family which also includes IL-17B, C, D, and E. These pro-inflammatory cytokines bind to a heteromeric receptor complex composed of the IL-17 receptor A (IL-17RA) and the IL-17 receptor C (IL-17RC) to mediate host protection against infections, while also contributing to the pathogenesis of various autoimmune diseases. IL-17A and IL-17F share $50 \%$ sequence identity and are known to function as homodimers. Nevertheless, they can also form a biologically active heterodimer, IL-17AF, ${ }^{2-4}$ which also signals through the IL-17RA/IL-17RC receptor complex, ${ }^{5}$ but whose physiological role is still largely unknown. We have determined the crystal structure of human IL-17AF at $2.3 \AA$ resolution. Like IL-17A and F, IL-17AF is a disulfide-linked dimer, with the two protein chains associated in a parallel manner. Both subunits show the expected cystine-knot fold with two intrachain disulfide bridges. The core of the dimer interface is mainly defined by one pair of anti-parallel $\beta$-strands from each chain and involves many hydrophobic residues conserved across IL-17A and F. Additionally, the $\mathrm{N}$-terminal segments of each protein chain reach the other subunit across the dimer interface, thus providing extensive additional contacts. Besides their similar overall topology, the IL-17RA receptor binding sites are highly conserved between the hetero- and homo-dimeric forms. ${ }^{6-8}$ In human IL-17AF, however, a hydrophobic pocket that is essential for IL-17RA recognition is occluded in the IL-17F subunit by a phenylalanine residue (Phe18) of the N-terminal region of IL-17A. Interestingly, while this binding site is also occupied by two phenylalanine residues (Phe10 and Phe11) in the IL-17F homodimer it is freely accessible in the IL-17A homodimer. ${ }^{6,8}$ This hydrophobic lock may thus be an important structural element in modulating IL-17RA receptor binding and may contribute to the distinct binding affinities of this receptor for the different IL-17A/F isoforms. ${ }^{5}$ References 1 . Wei J \& Chen D (2013) Emerging Microbes \& Infections 2, e60. 2. Chang SH, Dong C (2007) Cell Res; 17(5): 435-40. 3. Liang SC et al (2007) J Immunol; 179: 7791-7799. 4. Wright JF et al (2007) J Biol Chem; 282: 13447-13455. 5. Wright JF et al (2008) J Immunol; 181: 2799-2805. 6. Hymowitz SG et al (2001) EMBO J; 20(19): 5332-41. 7. Ely LK et al (2009) Nat Immunol; 10(12): 1245-51. 8. Liu S et al (2013) Nat Commun; $4: 1888$.

Keywords: IL-17, cytokine, structure
MS7. Nucleic acids and their complexes and assemblies with proteins

Chairs: Miquel Coll, Christine Cardin

MS7-P1 Structural basis for the inhibition of CCL2-signaling by a mirror-image aptamer

Christian Betzel $^{1}$, Oberthuer Dominik ${ }^{1,2}$

1. Laboratory for Structural Biology of Infection and Inflammation, University of Hamburg, c/o DESY Building 22a, Notkestrasse 85, 22607 Hamburg, Germany

2. Center for Free-Electron Laser Science, Deutsches Elektronen Synchrotron-DESY, Notkestrasse 85, 22607 Hamburg, Germany

email: Christian.Betzel@uni-hamburg.de

The crystal structure of a 40mer mirror-image RNA oligonucleotide completely built from nucleotides of the non-natural L-chirality in complex with the pro-inflammatory chemokine L-CLL2 (monocyte chemoattractant protein 1), a natural protein composed of regular L-amino acids, will be reported (Oberthuer, 2014). The L-oligonucleotide is an L-aptamer (a Spiegelmer®) identified to bind L-CCL2 with high affinity, thereby neutralizing the chemokine's activity. CCL2 plays a key role in attracting and positioning monocytes; its overexpression in several inflammatory diseases makes CCL2 an interesting pharmacological target. The PEGylated form of the L-aptamer, NOX-E36 (emapticap pegol), already showed promising efficacy in clinical Phase II studies conducted in diabetic nephropathy patients. The structure of the Loligonucleotide $\cdot$ L-protein complex was solved and refined to $2.05 \AA$. It unveils the L-aptamer's intramolecular contacts and permits, in combination with binding studies, a detailed analysis of its structure-function relationship. Furthermore, the analysis of the intermolecular drug-target interactions reveals insight into the selectivity of the L-aptamer for certain related chemokines. Oberthuer, D. et al. Crystal structure of a mirror-image L-RNA aptamer (Spiegelmer) in complex with the natural L-protein target CCL2. Nat Commun 6, 6923 (2015).

Keywords: CCL2, aptamers, L-RNA, chemokines, drug-target interactions 